\title{
ON DIFFERENTIABILITY OF PEANO TYPE FUNCTIONS. III
}

\author{
JACEK CICHOŃ AND MICHA€ MORAYNE
}

\begin{abstract}
We show that for all positive natural numbers $m, n$ the following two sentences are equivalent: (i) $2^{\aleph_{0}} \leq \aleph_{n}$; (ii) there exists an onto function $f: R^{n} \rightarrow R^{n+m}$ ( $R$ the set of real numbers) such that at each point of $R^{n}$ at least $n$ coordinates of $f$ are differentiable.
\end{abstract}

0. Introduction. For each natural number $n \geq 1$ we present, using notions of classical mathematical analysis, a sentence $\varphi_{n}$ equivalent to the sentence $2^{\aleph_{0}} \leq \aleph_{n}$.

The last sentence is independent of ZFC. R. Solovay has shown that $2^{\aleph_{0}}$ can be any cardinal number the cofinality of which is uncountable (for a precise formulation of this result see [8]). In particular, for each natural number $n \geq 1$ the theory $\mathrm{ZFC}+2^{\aleph_{0}}=\aleph_{n}$ is consistent (whenever the theory ZFC is consistent). Solovay's result implies as well that the theory ZFC $+2^{\aleph_{0}}>\aleph_{\omega}$ is consistent ( $\omega$ denotes here the order type of the set of all natural numbers). The sentence $2^{\aleph_{0}}=\aleph_{1}$ is called the Continuum Hypothesis, and its independence of ZFC results from the work of K. Gödel and P. J. Cohen.

The sentence $\varphi_{n}$ proclaims that there exists an onto function $f: R^{n} \rightarrow R^{n+m}$ ( $R$ denotes the set of real numbers), where $m$ is any natural number $\geq 1$, such that at each point of $R^{n}$ at least $n$ coordinates of $f$ are differentiable.

We also analyse the question concerning regularity of such functions.

This paper is a continuation of $[\mathbf{3}$ and $\mathbf{4}]$. Our results generalize theorems from those papers in which the existence of onto functions $f: R \rightarrow R^{n}$, where at each point of $R$ at least one coordinate of $f$ is differentiable, is analysed.

1. Auxiliary theorems. Let $R$ denote the set of real numbers and $N$ the set of natural numbers, where $i, j, k, l, m, n, p, r \in N$. For any sets $A$ and $B$ the set of all functions from $A$ to $B$ is denoted by $B^{A}$. We treat the cartesian product $A^{n}$ as the set $A^{\{1, \ldots, n\}}$. This convention enables us to consider $k$-dimensional sections of subsets of $R^{n}$ more easily.

The cardinality of a set $X$ is denoted by $|X|$.

Let $P_{n}^{m}=[\{1, \ldots, m\}]^{n}$ be the set of all subsets of $\{1, \ldots, m\}$, the cardinality of which is $n$. For a fixed product $X^{m}$ and $A \subseteq X^{m}, \Lambda \in P_{n}^{m}, a \in X^{m}$ (or $a \in X^{\Lambda}$ ), let $(A)_{a, \Lambda}=\{x \in A: x(i)=a(i)$ for each $i \in \Lambda\}$.

A family $\left\{E_{\Lambda}: \Lambda \in P_{n}^{m}\right\}$ is called the $(m, n)$-Sierpiński partition of a set $X^{m}$ if $\bigcup\left\{E_{\Lambda}: \Lambda \in P_{n}^{m}\right\}=X^{m}$ and, for every $\Lambda \in P_{n}^{m}$ and $a \in X^{m}$, we have $\left|\left(E_{\Lambda}\right)_{a, \Lambda}\right| \leq \aleph_{0}$. W. Sierpiński $[6]$ investigated the existence of such partitions of $R^{m}$ for small natural numbers $m, n>0$. A general theorem about the existence of $(m, n)$ -

Received by the editors April 25, 1983.

1980 Mathematics Subject Classification. Primary 04A30, $26 \mathrm{~B} 05$.

Key words and phrases. Continuum hypothesis, differentiability, Peano function, Lipschitz condition. 
Sierpiński partitions of $X^{m}$ for an arbitrary set $X$ was proved by R. Sikorski [7, p. 18]. We shall use a special case of this theorem.

THEOREM 1.1. Let $m, n$ be arbitrary natural numbers such that $m>n$ and let $X$ be any set. Then the following sentences are equivalent:

(1) $|X| \leq \aleph_{n}$

(2) the $(m, n)$-Sierpinski partition of $X^{m}$ exists.

Let $\lambda_{n}$ be Lebesgue measure in $R^{n}$. Let $\left(\lambda_{n}\right)_{*}$ and $\left(\lambda_{n}\right)^{*}$ denote inner and outer Lebesgue measure in $R^{n}$ respectively.

Let $L_{n}$ be the ideal of subsets of $R^{n}$ of Lebesgue measure zero.

We shall use the following theorem proved by H. G. Eggleston [1].

THEOREM 1.2. Let $A$ be a Lebesgue measurable subset of $R^{n}$ such that $\lambda_{n}(A)>$ 0 . Then there are nonempty perfect sets $P_{1}, \ldots, P_{n} \subseteq R$ such that $P_{1} \times \cdots \times P_{n} \subseteq A$.

A subset $A$ of $R^{n}$ is an analytic set if there exists a continuous function $f: R \rightarrow$ $R^{n}$ and a Borel set $B \subseteq R$ such that $A=f(B)$.

It is known that every analytic set is Lebesgue measurable (see [2, Chapter XII, $\S 8$, Theorem 7a, p. 431]).

We shall need the following theorem of Mazurkiewicz (see [2, Chapter XIV, 3 , Theorem 3, p. 468]).

THEOREM 1.3. If $B \subseteq R^{n}$ is a Borel set and $f: B \rightarrow R^{m}$ is continuous there exists a coanalytic (the complement of an analytic set) selector of the family $\left\{f^{-1}(\{y\}): y \in f(B)\right\}$.

2. The Banach condition $T_{2}$. Now our aim is to prove Corollary 2.7, this being a generalization of the well-known fact for the one-dimensional case.

DEFinition 2.1. Let $A \subseteq R^{n}$ and $f: R^{n} \rightarrow R^{m}$.

(1) $f$ satisfies the Lipschitz condition on $A(f \in L(A))$ if there exists a constant $C$ such that for every $x, y \in A$ we have $|f(x)-f(y)| \leq C|x-y|$.

(2) $f$ satisfies the generalized Lipschitz condition on $A(f \in L G(A))$ if there exists a family of sets $\left\{A_{k}: k \in N\right\}$ such that $\bigcup\left\{A_{k}: k \in N\right\}=A$ and, for every $k \in N$, we have $f \in L\left(A_{k}\right)$.

(3) $f$ is differentiable at a point $x \in R^{n}(f \in \operatorname{Diff}(x))$ if there exists a linear mapping $B: R^{n} \rightarrow R^{m}$ such that $|f(x+h)-f(x)-B(h)| /|h| \rightarrow 0$ when $|h| \rightarrow 0$;

(4) $f$ is differentiable on $A(f \in \operatorname{Diff}(A))$ if $f \in \operatorname{Diff}(x)$ for each $x \in A$.

LEMMA 2.2. If $A \subseteq R^{n}$ and $f: R^{n} \rightarrow R^{m}$ then $f \in \operatorname{Diff}(A)$ implies $f \in L G(A)$.

Proof. For each $x \in A$ there exists a linear mapping $B_{x}: R^{n} \rightarrow R^{m}$ such that $\left|f(x+h)-f(x)-B_{x}(h)\right| /|h| \rightarrow 0$ when $|h| \rightarrow 0$. Hence, for each $x$ we can find $\varepsilon(x)>0$ such that if $|h|<\varepsilon(x)$ then $|f(x+h)-f(x)| \leq|h|\left(\left\|B_{x}\right\|+1\right)$, where $\|\cdot\|$ stands for the standard norm of a linear mapping, i.e.

$$
\|B\|=\sup \left\{|B(a)| /|a|: a \in R^{n}-\{0\}\right\} .
$$

Let us put

$$
S_{n}=\left\{x \in A:\left\|B_{x}\right\|<n\right\}, \quad T_{m}=\{x \in A: \varepsilon(x)>1 / m\} .
$$


We can take a sequence of nonoverlapping cubes $Q_{k}^{(m)}$ of diameter less than $1 / m$ such that $R^{n}=\bigcup\left\{Q_{k}^{(m)}: k \in N\right\}$. One can easily see that $f \in L\left(S_{n} \cap T_{m} \cap Q_{k}^{(m)}\right)$ for each $k, m, n \in N$ and $A=\bigcup\left\{S_{n}: n \in N\right\}=\bigcup\left\{T_{m}: m \in N\right\}$. Hence $f \in L G(A)$.

DEFINITION 2.3. A function $f: R^{n} \rightarrow R^{m}$ satisfies the Lusin condition on a set $A \subseteq R^{n}$ if $N \in L_{n}$ implies $f(N) \in L_{m}$ for every $N \subseteq A$.

Notice that if $A \subseteq R^{n}, f: R^{n} \rightarrow R^{n}$ and $f \in L G(A)$, then $f$ satisfies the Lusin condition on $A$.

DEFinition 2.4. The function $f: R^{n} \rightarrow R^{n}$ satisfies the Banach condition $T_{2}$ on a set $A \subseteq R^{n}\left(f \in T_{2}(A)\right)$ if $\left\{y \in f(A):\left|f^{-1}(\{y\}) \cap A\right|>\aleph_{0}\right\} \in L_{n}$.

LEMMA 2.5. If a function $f: R^{n} \rightarrow R^{n}$ satisfies the Lipschitz condition on a set $A \subseteq R^{n}$ then $f \in T_{2}(A)$.

Proof. If $f \in L(A)$ we can extend continuously the function $f \mid A$ ( $f$ restricted to $A)$ to the function satisfying the Lipschitz condition on the closure of $A$, and therefore we can assume $A$ is a closed set. Let us consider the family $C=\left\{f^{-1}(y) \cap A: y \in R^{n}\right\}$ and a maximal family $D$ of pairwise disjoint Borel measurable subselectors (i.e. subsets of selectors) of positive measure for $C$. Obviously, $D$ is countable, hence the set $A-\bigcup D$ is Borel measurable. By Theorem 1.3 one can find a coanalytic, hence Lebesgue measurable, selector $S$ for the family $\left\{f^{-1}(\{y\}) \cap(A-\bigcup D): y \in R^{n}\right\}$. If $\lambda_{n}(S)>0$ then there exists a closed subset of $S$ of positive measure which is, of course, a subselector for $C$ disjoint with UD, and this contradicts the assumption that $D$ is maximal. Thus $\lambda_{n}(S)=0$. It can easily be seen, regarding the countability of $D$, that $S$ includes the selector $S_{1}$ for the family $\left\{f^{-1}(\{y\}) \cap A: y \in Y\right\}$, where $Y=\left\{y:\left|f^{-1}(\{y\}) \cap A\right|>\aleph_{0}\right\}$. Obviously, $\lambda_{n}\left(S_{1}\right)=0$ and $f\left(S_{1}\right)=Y$. But $f \in L(A)$; hence $f$ satisfies the Lusin condition on $A$. Thus $\lambda_{n}(Y)=0$ and, by definition, $f \in T_{2}(A)$.

From the above lemmas we obtain the following corollaries:

Corollary 2.6. If $f: R^{n} \rightarrow R^{n}, A \subseteq R^{n}$ and $f \in L G(A)$, then $f \in T_{2}(A)$.

Corollary 2.7. If $f: R^{n} \rightarrow R^{n}, A \subseteq R^{n}$ and $f \in \operatorname{Diff}(A)$, then $f \in T_{2}(A)$.

The above corollary for the case $n=1$ when $A$ is an interval follows from Theorem 10.1, Chapter VII, p. 234 and Theorem 6.3, Chapter IX, p. 279 in [5].

3. The main result. We prove the theorem announced in the introduction.

DEFINITION 3.1. Let $f=\left(f_{1}, \ldots, f_{m}\right): R^{n} \rightarrow R^{m}$.

(1) If $\Lambda=\left\{i_{1}, \ldots, i_{k}\right\} \subseteq\{1, \ldots, m\}$ then $f_{\Lambda}$ is the function defined by $f_{\Lambda}=$ $\left(f_{i_{1}} \ldots, f_{i_{k}}\right)$. We consider this function as a mapping from $R^{n}$ to $R^{\Lambda}$.

(2) $f$ is $k$-differentiable if for each $x \in R^{n}$ there exists $\Lambda \in P_{k}^{m}$ such that $f_{\Lambda} \in \operatorname{Diff}(x)$

(3) $f$ is $k$-measurable if there exists $\Lambda \in P_{k}^{m}$ such that $f_{\Lambda}$ is a Lebesgue measurable mapping from $R^{n}$ to $R^{\Lambda}$.

THEOREM 3.2. For arbitrary $m, n>0$ and $k \geq 0$ the following sentences are equivalent:

(1) $2^{\aleph_{0}} \leq \aleph_{n-k}$;

(2) there exists a function $f: R^{n} \rightarrow R^{n+m}$ onto $R^{n+m}$ which is $n$-differentiable and $k$-measurable. 
PROOF. (1) $\rightarrow(2)$. Hence, assume that $2^{\aleph_{0}} \leq \aleph_{n-k}$. Notice that we can assume $k<n$.

Let $K=n-k$ and $L=n+m-k$.

First we prove there exists an onto function $h: R^{K} \rightarrow R^{L}$ which is $K$-differentiable. Having this function we shall easily construct the needed function $f$.

Let us fix $L$ different positive real numbers $q_{1}, \ldots, q_{L}$. Let

$$
\Phi_{i}\left(x_{1}, \ldots, x_{K}\right)=\left(\left(q_{i}\right)^{1} \cdot x_{1}+\cdots+\left(q_{i}\right)^{K} \cdot x_{K}\right) \sin \left(\left(q_{i}\right)^{1} \cdot x_{1}+\cdots+\left(q_{i}\right)^{K} \cdot x_{K}\right)
$$

and $\Phi=\left(\Phi_{1}, \ldots, \Phi_{L}\right)$. Then $\Phi: R^{K} \rightarrow R^{L}$.

For an arbitrary sequence of integers $i_{1}, \ldots, i_{K}$, put

$$
A_{i_{1}, \ldots, i_{K}}=\left[4 \pi i_{1}, 4 \pi i_{1}+2 \pi\right] \times \cdots \times\left[4 \pi i_{K}, 4 \pi i_{K}+2 \pi\right] .
$$

Let $\left\{\Lambda_{1}, \ldots, \Lambda_{T}\right\}$ be an enumeration of all elements of $P_{K}^{L}$. Put

$$
B_{\Lambda_{j}}=\bigcup\left\{A_{i_{1}, \ldots, i_{K}}: i_{1}+\cdots+i_{K} \equiv j(\bmod T)\right\} .
$$

The function $\Phi$ is differentiable and $\left\{B_{\Lambda}: \Lambda \in P_{K}^{L}\right\}$ is a family of closed and pairwise disjoint sets. The following claim formulates an important property of $\Phi$.

Claim 1. For sufficiently large $q_{1}, \ldots, q_{L}$ and for every $\Lambda \in P_{K}^{L}$ and each $y \in R^{K}$ the set $\left\{x \in B_{\Lambda}: \Phi_{\Lambda}(x)=y\right\}$ is infinite.

Let $\Lambda=\left\{i_{1}, \ldots, i_{K}\right\}$. We consider a new coordinate system:

$$
\begin{aligned}
& z_{1}=\left(q_{i_{1}}\right)^{1} \cdot x_{1}+\cdots+\left(q_{i_{1}}\right)^{K} \cdot x_{K} \\
& \vdots \\
& z_{K}=\left(q_{i_{K}}\right)^{1} \cdot x_{1}+\cdots+\left(q_{i_{K}}\right)^{K} \cdot x_{K} .
\end{aligned}
$$

The new axes are defined correctly, for

$$
\operatorname{det}\left[\begin{array}{ccc}
\left(q_{i_{1}}\right)^{1} & \cdots & \left(q_{i_{1}}\right)^{K} \\
\vdots & & \\
\left(q_{i_{K}}\right)^{1} & \cdots & \left(q_{i_{K}}\right)^{K}
\end{array}\right]=\prod_{j<l}\left(q_{i_{j}}-q_{i_{l}}\right) \cdot \prod_{j=1}^{K} q_{i_{j}} \neq 0 .
$$

We call a cube with edges of length $2 \pi$ parallel to the axes $O Z_{1}, \ldots, O Z_{K}$ a standard $Z$-cube.

Multiplying the numbers $q_{1}, \ldots, q_{L}$ by a sufficiently large constant, we can ensure that, for each sequence of integers $i_{1}, \ldots, i_{K}$, the set $A_{i_{1}, \ldots, i_{K}}$ contains a standard $Z$ cube. Thus there are standard $Z$-cubes with arbitrarily large coordinates contained in the set $B_{\Lambda}$.

Let $y=\left(y_{i_{1}}, \ldots, y_{i_{K}}\right)$. For a standard $Z$-cube $S$ with sufficiently large coordinates we can choose $\left(z_{1}, \ldots, z_{K}\right) \in S$ such that $z_{1} \sin z_{1}=y_{i_{1}}, \ldots, z_{K} \sin z_{K}=$ $y_{i_{K}}$. Thus the set $\left\{x \in B_{\Lambda}: \Phi_{\Lambda}(x)=y\right\}$ is infinite and the claim is proved.

We now define the desired function $h: R^{K} \rightarrow R^{L}$. On the strength of the assumption $2^{\aleph_{0}} \leq \aleph_{K}$ and by Theorem 1.1, there exists an $(L, K)$-Sierpiński partition $\left\{E_{\Lambda}: \Lambda \in P_{K}^{L}\right\}$ of $R^{L}$. For $a \in R^{\Lambda}$ let $\left\{y_{0, \Lambda}^{a}, y_{1, \Lambda}^{a}, \ldots\right\}=\left(E_{\Lambda}\right)_{a, \Lambda}$. By Claim 1 we can choose a sequence $\left\{x_{0, \Lambda}^{a}, x_{1, \Lambda}^{a}, \ldots\right\}$ of different elements from the set $\left\{x \in B_{\Lambda}: \Phi_{\Lambda}(x)=a\right\}$. We put $h\left(x_{i, \Lambda}^{a}\right)=y_{i, \Lambda}^{a}$ for each $i$. For every $x \in R^{K}$ for which the value $h(x)$ has not been so defined, we put $h(x)=\Phi(x)$. 
Notice at first that $h$ is a surjection onto $R^{L}$. In order to see this let $y \in R^{L}$. Then for some $\Lambda \in P_{K}^{L}$ we have $y \in E_{\Lambda}$. Let $a=(y)_{\Lambda}$ be the projection of $y$ onto $R^{\Lambda}$. Then there exists $i$ such that $y=y_{i, \Lambda}^{a}$. Hence $h\left(x_{i, \Lambda}^{a}\right)=y$.

Note that if $x \in R^{K}-\bigcup\left\{B_{\Lambda}: \Lambda \in P_{K}^{L}\right\}$ then $h(x)=\Phi(x)$, and if $x \in B_{\Lambda}$ then $h_{\Lambda}(x)=\Phi_{\Lambda}(x)$. Thus $h$ is $K$-differentiable.

We now show how to reconstruct the function $h$ to obtain a function $f: R^{n} \rightarrow$ $R^{n+m}$, the existence of which is postulated by sentence (2) of the theorem.

In fact it is enough to put

$$
\begin{aligned}
& f\left(x_{1}, \ldots, x_{k}, x_{k+1}, \ldots, x_{n}\right) \\
& \quad=\left(x_{1}, \ldots, x_{k}, h_{1}\left(x_{k+1}, \ldots, x_{n}\right), \ldots, h_{n+m-k}\left(x_{k+1}, \ldots, x_{n}\right)\right) .
\end{aligned}
$$

It is self-evident that $f$ has the desired properties. This completes the proof of $(1) \rightarrow(2)$.

We now prove $(2) \rightarrow(1)$. Let $f=\left(f_{1}, \ldots, f_{n+m}\right): R^{n} \rightarrow R^{n+m}$ be an $n$-differentiable and $k$-measurable surjection onto $R^{n+m}$. We assume that the coordinates $f_{1}, \ldots, f_{k}$ are measurable. Of course we can also assume $k \leq n$.

For $\Lambda \in P_{n}^{n+m}$ let $D_{\Lambda}=\left\{x \in R^{n}: f_{\Lambda} \in \operatorname{Diff}(x)\right\}$. By the assumption of $n$ differentiability of $f$ we have $R^{n}=\bigcup\left\{D_{\Lambda}: \Lambda \in P_{n}^{n+m}\right\}$.

Let $A=\left\{\Lambda \in P_{n}^{n+m}:\{1, \ldots, k\} \nsubseteq \Lambda\right\}$ and $B=\left\{\Lambda \in P_{n}^{n+m}:\{1, \ldots, k\} \subseteq \Lambda\right\}$.

Claim 2. If $\Lambda \in \mathcal{A}$ then $f\left(D_{\Lambda}\right) \in L_{n+m}$.

Let $\Lambda \in A$ and $i \in\{1, \ldots, k\}-\Lambda$. By Lemma $2.2 f_{\Lambda} \in L G\left(D_{\Lambda}\right)$. Hence there exists a partition $\left\{A_{r}: r \in N\right\}$ of $D_{\Lambda}$ such that $f_{\Lambda} \in L\left(A_{r}\right)$ for each $r \in N$. Let $f_{\Lambda, r}$ be an extension of $f_{\Lambda} \mid A_{r}$ to a continuous function on $\bar{A}_{r}$. It is easy to see that such an extension exists and that $f_{\Lambda, r} \in L\left(\bar{A}_{r}\right)$.

By Lebesgue measurability of $f_{i}$ and Lusin's theorem (see [5, Theorem 7.1, Chapter III, p. 72]), we can express $R^{n}$ as the sum $R^{n}=\bigcup\left\{B_{p}: p \in N\right\} \cup N$, where $B_{p}$ are closed sets and $f_{i}$ is continuous on $B_{p}$ for each $p \in N$ and $N \in L_{n}$. Let $C_{r}^{p}=\bar{A}_{r} \cap B_{p}$ and $D_{r}=A_{r} \cap N$. The set $C_{r}^{p}$ is closed and the function $\left(f_{\Lambda, r}, f_{i}\right)$ is continuous on $C_{r}^{p}$; therefore the set $E_{r}^{p}=\left(f_{\Lambda, r}, f_{i}\right)\left(C_{r}^{p}\right)$ is a Borel set and, hence, Lebesgue measurable.

By Corollary 2.6 applied to $f_{\Lambda, r}$, there exists a set $S \subseteq R^{\Lambda}$ of Lebesgue measure zero such that, for $y \in R^{\Lambda}-S$, the set $f_{\Lambda, r}^{-1}(\{y\}) \cap C_{r}^{p}$ is countable. Thus the set $\left\{z \in R:(y, z) \in E_{r}^{p}\right\}$ is also countable. Hence, by Fubini's theorem, $E_{r}^{p} \in L_{n+1}$. This holds for each natural $p$ and $r$, whence $E=\bigcup_{p} \bigcup_{r} E_{r}^{p} \in L_{n+1}$.

For each $r \in N$ we have $f_{\Lambda} \in L\left(A_{r}\right)$, whence $f_{\Lambda}$ satisfies the Lusin condition on $A_{r}$ and, therefore, $F=\bigcup_{r}\left(f_{\Lambda}, f_{i}\right)\left(D_{r}\right) \in L_{n+1}$.

Hence, $\left(f_{\Lambda}, f_{i}\right)\left(D_{\Lambda}\right) \subseteq E \cup F \in L_{n+1}$ and, furthermore, $f\left(D_{\Lambda}\right) \in L_{n+m}$. This completes the proof of Claim 2 .

Let $T_{\Lambda}=\left\{y \in f_{\Lambda}\left(D_{\Lambda}\right):\left|f_{\Lambda}^{-1}(\{y\}) \cap D_{\Lambda}\right|>\aleph_{0}\right\}$ for $\Lambda \in B$. By definition of $D_{\Lambda}$ we have $f_{\Lambda} \in \operatorname{Diff}\left(D_{\Lambda}\right)$. Hence, by Corollary 2.7, $f_{\Lambda} \in T_{2}\left(D_{\Lambda}\right)$ and therefore $T_{\Lambda} \in L_{n}$.

Let $T=\bigcup\left\{T_{\Lambda} \times R^{\{1, \ldots, n+m\}-\Lambda}: \Lambda \in B\right\} \cup f\left(\bigcup\left\{D_{\Lambda}: \Lambda \in A\right\}\right)$. By the above remark and Claim 2 we obtain $T \in L_{n+m}$. By virtue of Theorem 1.2 we can find nonempty perfect sets $P_{1}, \ldots, P_{n+m}$ such that $P_{1} \times \cdots \times P_{n+m} \subseteq R^{n+m}-T$.

Let $\left(p_{1}, \ldots, p_{k}\right) \in P_{1} \times \cdots \times P_{k}$ and let

$$
P^{\prime}=\left\{p_{1}\right\} \times \cdots \times\left\{p_{k}\right\} \times P_{k+1} \times \cdots \times P_{n+m} \quad \text { and } \quad P=P_{k+1} \times \cdots \times P_{n+m} .
$$


For $\Lambda \in P_{n-k}^{n+m-k}$ we put

$$
E_{\Lambda}^{\prime}=P^{\prime} \cap f\left(D_{\{1, \ldots, k\} \cup\{i+k: i \in \Lambda\}}\right) \quad \text { and } \quad E_{\Lambda}=\left\{x \in P:\left(p_{1}, \ldots, p_{k}, x\right) \in E_{\Lambda}^{\prime}\right\} .
$$
of $P$.

We shall show that $\left\{E_{\Lambda}: \Lambda \in P_{n-k}^{n+m-k}\right\}$ is a $(m+n-k, n-k)$-Sierpiński partition

Let $a \in P$ and $\Lambda \in P_{n-k}^{n+m-k}$. Let $x \in\left(E_{\Lambda}\right)_{a, \Lambda}$. Then $x \in E_{\Lambda}$ and for each $i \in \Lambda$ we have $x_{i}=a_{i}$. One can see that

$$
\left(p_{1}, \ldots, p_{k}, x\right) \in f\left(D_{\{1, \ldots, k\} \cup\{i+k: i \in \Lambda\}}\right)
$$

and

$$
\left(p_{1}, \ldots, p_{k}, x\right) \notin T_{\{1, \ldots, k\} \cup\{i+k: i \in \Lambda\}} \times R^{\{1, \ldots, n+m\}-(\{1, \ldots, k\} \cup\{i+k: i \in \Lambda\})} .
$$

It is easy to check that there are only countably many such $x$ 's. By Theorem 1.1 we have $2^{\aleph_{0}} \leq \aleph_{n-k}$. Hence the theorem is proved.

\section{Discussion.}

1. One can easily see that in the proof of the implication $(2) \rightarrow(1)$ we use only the fact that $\left(\lambda_{n+m}\right)_{*}\left(f\left(R^{n}\right)\right)>0$. Thus we can formulate the following theorem.

THEOREM $3.2^{\prime}$. For $m, n>0$ and $k \geq 0$ the following statements are equivalent:

(1) $2^{\aleph_{0}} \leq \aleph_{n-k}$;

$\left(2^{\prime}\right)$ there exists an $n$-differentiable and $k$-measurable function $f: R^{n} \rightarrow R^{n+m}$ such that $\left(\lambda_{n+m}\right)_{*}\left(f\left(R^{n}\right)\right)>0$.

In the construction of $f$ in the proof of Theorem 3.2 we in fact construct the function which is $n$-analytic (i.e. for each point $x$ one can choose $n$ coordinate functions analytic at $x$ ) and has $k$ analytic coordinate functions. So we can also give the following version of Theorem 3.2.

THEOREM $3.2^{\prime \prime}$. For $m, n>0$ and $k \geq 0$ the following statements are equivalent:

(1) $2^{\aleph_{0}} \leq \aleph_{n-k}$

$\left(2^{\prime \prime}\right)$ there exists an onto function $f: R^{n} \rightarrow R^{n+m}$ which is $n$-analytic and has at least $k$ analytic coordinate functions.

2. One cannot construct an onto function $f: R^{n} \rightarrow R^{n+m}$ which is $(n+1)$ differentiable because of the following theorem.

THEOREM 4.1. If $m, n>0$ and $f: R^{n} \rightarrow R^{n+m}$ is $(n+1)$-differentiable then $\lambda_{n+m}\left(f\left(R^{n}\right)\right)=0$.

Proof. For $\Lambda \in P_{n+1}^{n+m}$ let $D_{\Lambda}=\left\{x: f_{\Lambda} \in \operatorname{Diff}(\{x\})\right\}$. By assumption

$$
\bigcup\left\{D_{\Lambda}: \Lambda \in P_{n+1}^{n+m}\right\}=R^{n} \text {. }
$$

We shall show that $f\left(D_{\ell}\right) \in L_{r \dashv n}$.

Let $g\left(x_{1}, \ldots, x_{n+1}\right)=f_{\Lambda}\left(x_{1}, \ldots, x_{n}\right)$. Then $g$ is differentiable on the set $D_{\Lambda} \times R$. Hence $g \in L G\left(D_{\Lambda} \times R\right)$ and therefore satisfies the Lusin condition on $D_{\Lambda} \times R$. Obviously, $D_{\Lambda} \times\{0\} \subseteq D_{\Lambda} \times R$ and $D_{\Lambda} \times\{0\} \in L_{n+1}$. Hence,

$$
f_{\Lambda}\left(D_{\Lambda}\right)=g\left(D_{\Lambda} \times\{0\}\right) \in L_{n+1} \text {. }
$$

Thus $f_{\Lambda}\left(D_{\Lambda}\right) \in L_{n+m}$ and this completes the proof of the theorem.

If we assume $f: R^{n} \rightarrow R^{n+m}$ is $(n-1)$-differentiable, we can formulate the following theorem. 
THEOREM 4.2. For arbitrary $m, n>0$ there exists a continuous onto function $f: R^{n} \rightarrow R^{n+m}$ which has $(n-1)$-analytic coordinate functions.

PROOF. Let $g$ be a continuous function mapping $R$ onto $R^{m+1}$ (i.e. a Peano function). Let $f\left(x_{1}, \ldots, x_{n}\right)=\left(x_{1}, \ldots, x_{n-1}, g\left(x_{n}\right)\right)$. It is obvious that $f$ has the desired properties.

3. In Theorem $3.2^{\prime}$ the inner Lebesgue measure appears. A natural question arises about the outer Lebesgue measure of the image of the functions considered in this paper. The following theorem (together with Theorems 4.1 and 4.2) provides an answer to this question.

THEOREM 4.3. For $m, n>0$ there exists a function $f: R^{n} \rightarrow R^{n+m}$ such that $n$ coordinate functions of $f$ are analytic and $f\left(R^{n}\right)$ has full outer measure (i.e. for any measurable set $P \subseteq R^{n+m}$ of finite measure we have $\left(\lambda_{n+m}\right)^{*}\left(f\left(R^{n}\right) \cap P\right)=$ $\lambda_{n+m}(P)$.

PROOF. Let $g: R^{n} \rightarrow R^{m}$ be a function which has a graph with full outer Lebesgue measure. Such a function can be constructed in the following way:

Let $\left\{F_{\xi}\right\}_{\xi<2^{\aleph_{0}}}$ be an enumeration of all Borel subsets of positive measure of $R^{n+m}$. By transfinite induction we construct the sequence of points $\left\{\left(x_{\xi}, y_{\xi}\right)\right\}_{\xi<2^{\aleph_{0}}}$ such that for each $\xi<2^{\aleph_{0}}$ we have $\left(x_{\xi}, y_{\xi}\right) \in F_{\xi}$ and $x_{\eta} \neq x_{\xi}$ for $\eta<\xi$.

Put $g\left(x_{\xi}\right)=y_{\xi}$ for each $\xi<2^{\aleph_{0}}$. For the other $x$ 's $\in R^{n}$ put $g(x)=0$.

Let $f\left(x_{1}, \ldots, x_{n}\right)=\left(x_{1}, \ldots, x_{n}, g\left(x_{1}, \ldots, x_{n}\right)\right)$. It is obvious that $f\left(R^{n}\right)=$ $\operatorname{graph}(g)$ and, hence, $f\left(R^{n}\right)$ has full outer Lebesgue measure in $R^{n+m}$.

\section{REFERENCES}

1. H. G. Eggleston, Two measure properties of Cartesian product sets, Quart. J. Math. Oxford Ser. (2) 5 (1954), 108-115.

2. K. Kuratowski and A. Mostowski, Set theory, North-Holland, Amsterdam and Warsaw, 1976.

3. M. Morayne, On differentiability of Peano type functions, Colloq. Math. (to appear).

4. __ On differentiability of Peano type functions. II, Colloq. Math. (to appear).

5. S. Saks, Theory of the integral, Monografie Mat., vol. 7, PWN, Warsaw, 1937.

6. W. Sierpiński, Sur quelques propositions concernant la puissance du continu, Fund. Math. 38 (1951), $1-13$.

7. R. Sikorski, A characterization of alephs, Fund. Math. 38 (1951), 18-22.

8. R. Solovay, Independence results in the theory of cardinals, Notices Amer. Math. Soc. 10 (1963), 595. Abstract \#63T-395.

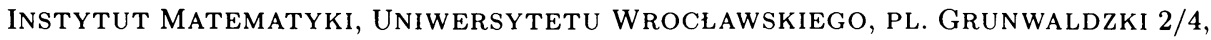
50384 WROCŁAW, POLAND 